\title{
TP53 mutation characteristics in therapy-related myelodysplastic syndromes and acute myeloid leukemia is similar to de novo diseases
}

\author{
Chi Young Ok1, Keyur P Patel', Guillermo Garcia-Manero², Mark J Routbort', Jie Peng1,3, Guilin Tang1, \\ Maitrayee Goswami ${ }^{1}$, Ken H Young ${ }^{1}$, Rajesh Singh ${ }^{1}$, L Jeffrey Medeiros ${ }^{1}$, Hagop M Kantarjian², \\ Rajyalakshmi Luthra ${ }^{1}$ and Sa A Wang ${ }^{1 *}$
}

\begin{abstract}
Background: TP53 mutation is more prevalent in therapy-related myeloid neoplasms (t-MN) than their de novo counterparts; however, the pattern of mutations involving TP53 gene in t-MN versus de novo diseases is largely unknown.

Methods: We collected 108 consecutive patients with therapy-related myelodysplastic syndrome (t-MDS)/acute myeloid leukemia (t-AML). Clinical, hematological, and cytogenetic data were collected by searching the electronic medical record. TP53 sequencing was performed in all patients using a clinically validated next-generation sequencing-based gene panel assay. A previously published patient cohort consisting of 428 patients with de novo MDS/AML was included for comparison.

Results: We assessed 108 patients with t-MN, in which 40 patients (37\%) had TP53 mutations. The mutation frequency was similar between t-MDS and t-AML; but significantly higher than de novo MDS/AML (62/428 patients, 14.5\%) $(p<0.0001)$. TP53 mutations in t-MN were mainly clustered in DNA-binding domains, with an allelic frequency of $37.0 \%$ (range, 7.1 to 98.8). Most mutations involved single nucleotide changes, of which, transitions (65.9\%) were more common than transversions (34.1\%). Missense mutations were the most frequent, followed by frameshift and nonsense mutations. This TP53 mutation pattern was strikingly similar to that observed in de novo MDS/AML. TP53 mutations in t-MN were associated with a complex karyotype $(p<0.0001)$, a higher number of chromosomal abnormalities $(p<0.0001)$, and an inferior overall survival in affected patients (6.1 vs 14.1 months) by univariate $(p<0.0001)$ and multivariate analyses $(p=0.0020)$.
\end{abstract}

Conclusions: Our findings support the recent notion that heterozygous TP53 mutation may be a function of normal aging and that mutated cells are subject to selection upon exposure to cytotoxic therapy. t-MN carrying TP53 mutation have an aggressive clinical course independent of other confounding factors.

Keywords: TP53, Therapy-related, MDS, AML, Karyotype

\section{Introduction}

Therapy-related myeloid neoplasms ( $\mathrm{t}-\mathrm{MN})$ are a group of hematopoietic myeloid neoplasms occurring in patients who previously received various cytotoxic chemotherapy regimens and/or radiation therapy for cancer, or rarely, non-neoplastic diseases [1]. Under the current WHO

\footnotetext{
* Correspondence: swang5@mdanderson.org

'Department of Hematopathology, The University of Texas MD Anderson

Cancer Center, 1515 Holcombe Boulevard, Houston, TX, USA

Full list of author information is available at the end of the article
}

classification, $\mathrm{t}-\mathrm{MN}$ is considered in a single category because these diseases invariably have a dismal outcome although morphologic variants, such as therapy-related myelodysplastic syndrome ( $\mathrm{t}$-MDS), therapy-related acute myeloid leukemia (t-AML), and therapy-related myelodysplastic syndrome/myeloproliferative neoplasm (t-MDS/ MPN) are recognized [2,3]. The incidence of $t-M N$ after chemotherapy and/or radiotherapy has been estimated to be $0.8 \%$ and $6.3 \%$ at 20 years [4]. t-MN are a group of clinically aggressive diseases and respond poorly to

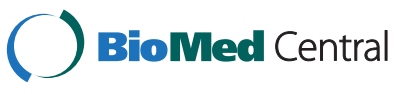


conventional therapies with rapid disease progression $[5,6]$. Chromosomal abnormalities are observed in up to $80 \%-90 \%$ t-MN patients with frequent high-risk cytogenetic abnormalities $[7,8]$. Our group recently showed that $\mathrm{t}-\mathrm{MN}$ has a mutational profile distinct from de novo MDS/AML [9].

p53 protein, encoded by TP53, is a tumor suppressor protein that consists of transactivation domain, prolinerich domain, DNA-binding domain, oligomerization domain, and regulatory domain [10]. p53 responds to diverse cellular stresses to induce cell cycle arrest, apoptosis, and DNA repair. Somatic TP53 mutations are found in a variety of cancers with various frequencies depending on cancer type [11]. Most TP53 mutations are clustered in the DNA-binding domain encompassing exons 5 and 8, and most mutations $(87.9 \%)$ in the DNA-binding domain are missense mutations [11]. Overall, TP53 mutations are found in 5\% 10\% of de novo MDS and AML and were shown to be associated with a complex karyotype and shorter survival [12-14]. In contrast, TP53 mutations are found in $21 \%-38 \% \mathrm{t}-\mathrm{MN}$ and are associated with $5 \mathrm{q}-$, a complex karyotype and a poor prognosis [15-17].

Recently, Wong and colleagues sequenced the genomes of 22 patients with $\mathrm{t}-\mathrm{AML}$ and showed that the total number of somatic single-nucleotide variants and the percentage of chemotherapy-related transversions were similar in t-AML and de novo AML [18]. These findings indicate that cytotoxic therapy does not induce genome-wide DNA damage, nor does cytotoxic therapy directly induce TP53 mutations. In four t-AML patients with TP53 mutation, the exact mutation in TP53 genes was found at a low frequency $(0.003 \%-0.7 \%)$ in mobilized blood leukocytes or bone marrow 3-6 years before the development of t-AML/t-MDS. Additionally, TP53 mutations at a low frequency were found in elderly healthy individuals. These findings indicate that TP53 mutations may be age related and that cells carrying this mutation might be resistant to chemotherapy and expand preferentially after treatment. The early acquisition of TP53 mutations in the founding stem cell clone probably contributes to the frequent cytogenetic abnormalities and poor responses to chemotherapy that are typical of patients with $\mathrm{t}-\mathrm{AML} / \mathrm{t}-\mathrm{MDS}$.

We conducted this study to compare the mutational characteristics of TP53 in t-MN and their de novo counterparts in a large patient cohort. We also correlated TP53 mutation status with the results of cytogenetic studies and evaluated the clinical significance of TP53 mutations in patients with $\mathrm{t}-\mathrm{MN}$.

\section{Results}

\section{Patient characteristics}

TP53 mutation analysis was performed on 108 patients' bone marrow (BM) whole cell samples, including 53
$\mathrm{t}$-MDS and $55 \mathrm{t}$-AML. The previous diseases in these patients included: 49 hematological malignancies, 45 carcinomas, 6 sarcomas, 5 germ cell tumors, 1 malignant mixed müllerian tumor, 1 medulloblastoma, and 1 rheumatoid arthritis. Sixty-one patients were treated with chemotherapy, 8 radiation therapy only and 39 with combined chemoradiation therapy. There were 62 men and 46 women with a median age of 68 years (range, 18-87). Karyotypic information was available in 105 patients and an abnormal karyotype was identified in $89(84.8 \%)$ patients, including 56 (53.3\%) with a complex karyotype ( $\geq 3$ abnormalities). The t-MDS cases could be further sub-categorized as refractory cytopenia with unilineage dysplasia (RCUD) $(n=$ $1,1.9 \%)$, refractory anemia with ring sideroblasts (RARS) $(n=1,1.9 \%)$, refractory cytopenia with multilineage dysplasia (RCMD) $(n=24,45.3 \%)$, refractory anemia with excess blasts (RAEB)-1 $(n=15,28.3 \%)$, and RAEB-2 $(n=12$, 22.6\%). According to the International Prognostic Scoring System (IPSS) risk categorization, 8 (15.7\%), 14 (27.5\%), and 29 (56.9\%) patients had good, intermediate, and poor cytogenetic risk, respectively. For 55 t-AML, 2 (3.7\%), 13 (24.1\%), and 39 (72.2\%) patients had favorable, intermediate, and adverse cytogenetic risk, respectively, using the revised United Kingdom Medical Research Council (UKMRC) prognostic system.

\section{TP53 mutations in t-MDS and t-AML}

A total of 47 different mutations in TP53 were detected in 40 of $108(37 \%)$ patients. Thirty-three $(n=33)$ had a single TP53 mutation, and 7 patients had two TP53 mutations. There were 41 single nucleotide changes and 6 insertion/deletions (indels). Among single nucleotide changes, there were 27 (65.9\%) transitions and 14 (34.1\%) transversions (Figure 1A). Classes of single nucleotide changes are described in Table 1 . The median mutational allelic burden was 37\% (range, 7.1\%-98.8\%) (Figure 1B). Twenty-nine (29) patients had mutations only involving TP53 and 11 patients had mutations in other genes including PTPN11, FLT3, IDH1, NRAS, KIT, JAK2, and MPL. Most TP53 mutations were missense $(n=38)$, followed by frameshift $(n=5)$ and nonsense $(n=4)$ mutations. Mutations were widely distributed in exons $4(n=2), 5(n=10)$, $6(n=12), 7(n=15), 8(n=7)$, and $10(n=1)$ (Figure 1C). Codon $248(n=7)$ was the most frequently mutated locus.

In t-MDS, 26 mutations in TP53 were identified in 21 of $53(39.6 \%)$ patients. The median mutational allelic burden was $34.9 \%$ (range, 10.4-91.7). There were 20 single nucleotide changes, of which transversions $(n=11)$ were more common than transitions $(n=9)$, and indels $(n=6)$. TP53 mutations in t-MDS were distributed in exons 5 $(n=8), 6(n=8), 7(n=6)$, and $8(n=4)$. In t-AML, 21 mutations in TP53 were detected in 19 of 55 (34.5\%) patients. The median mutational allelic frequency was $37.3 \%$ (range, 7.1-98.8), which was not significantly different 

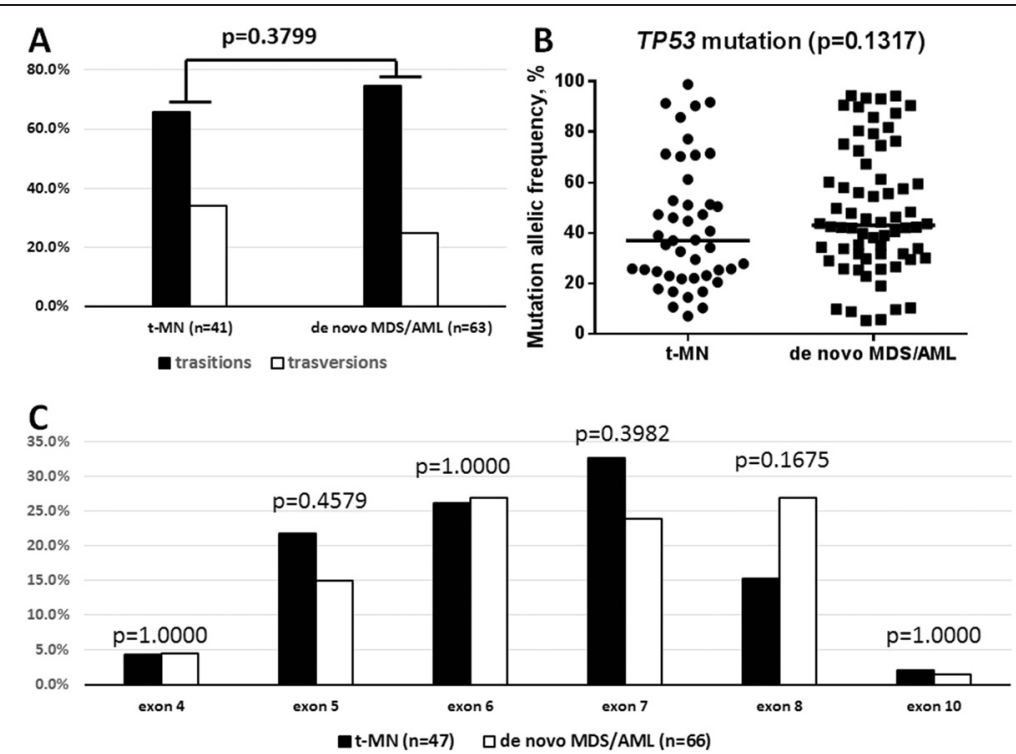

Figure 1 Patterns of TP53 mutations in t-MN and de novo MDS/AML. (A) Frequencies of transitions and transversions in t-MN and de novo MDS/ AML; the numbers in parentheses denote the number of single nucleotide changes in each group. (B) TP53 mutational allelic frequencies in t-MN and de novo MDS/AML. (C) Loci of TP53 mutations in t-MN and de novo MDS/AML. The numbers in parentheses denote the number of all mutations in each group.

from that of $\mathrm{t}$-MDS $(p=0.6624)$. All 21 mutations in t-AML were single nucleotide changes, of which 18 were transitions and 3 were transversions. The proportion of transversions was significantly lower in t-AML compared with $\mathrm{t}$-MDS $(11 / 20$ vs $3 / 21, p=0.0088)$. TP53 mutations in t-AML were detected in exons $4(n=2), 5(n=2), 6$ $(n=4), 7(n=9), 8(n=3)$, and $10(n=1)$, similar to t-MDS.

\section{Comparing TP53 mutations in t-MDS/AML and de novo MDS/AML}

We compared the current cohort with a previously published cohort [9] of de novo MDS/AML $(n=428)$ where TP53 mutation was detected in 62 (14.5\%) patients. In de novo MDS/AML, 56 patients had single TP53 mutation and 6 patients had double TP53 mutations. Similar to $\mathrm{t}$-MDS/AML, single nucleotide changes $(n=63)$ were the predominant form, followed by four indels and one duplication. Transitions were more frequent $(n=47,74.6 \%)$,

Table 1 Single nucleotide changes of TP53 mutations in therapy-related versus de novo myelodysplastic syndromes/acute myeloid leukemia (MDS/AML)

\begin{tabular}{lll}
\hline Class & $\mathbf{t}-\mathbf{M N}, \boldsymbol{n}$ (\%) & De novo MDS/AML, $\boldsymbol{n}(\%)$ \\
\hline $\mathrm{C}: \mathrm{G}>\mathrm{A}: \mathrm{T}$ & $5(12.2)$ & $1(1.6)$ \\
$\mathrm{C}: \mathrm{G}>\mathrm{G}: \mathrm{C}$ & $3(7.3)$ & $6(9.5)$ \\
$\mathrm{C}: \mathrm{G}>\mathrm{T}: \mathrm{A}$ & $18(43.9)$ & $35(55.6)$ \\
$\mathrm{T}: \mathrm{A}>\mathrm{A}: \mathrm{T}$ & $4(9.8)$ & $3(4.8)$ \\
$\mathrm{T}: \mathrm{A}>\mathrm{C}: \mathrm{G}$ & $9(22.0)$ & $12(19.0)$ \\
T:A > G:C & $2(4.9)$ & $6(9.5)$ \\
Total & $41(100)$ & $63(100)$ \\
\hline
\end{tabular}

similar to that in t-MDS/AML $(65.9 \%) \quad(p=0.3799)$ (Figure 1A). Classes of single nucleotide changes are described in Table 1. The median mutational allelic burden was $43.1 \%$ (range, 5.4-94.3), which was not significantly different from that of t-MDS/AML (37.0\%, $7.1 \%-98.8 \%)(p=0.1317)$ (Figure 1B). Similar to t-MDS/ AML, missense $(n=54)$ was the most common type of mutation, followed by nonsense $(n=7)$, frameshift $(n=5)$, and splice site mutations $(n=2)$. Mutations were detected in exons $4(n=3), 5(n=10), 6(n=18), 7 \quad(n=16), 8 \quad(n=$ $18)$, and $10(n=2)$ (Figure 1C). Comparing mutation distribution in different exons between $\mathrm{t}-\mathrm{MN}$ and de novo AML/MDS, significant difference was not observed in each exon. Fifty-two patients had mutations only in TP53 and 10 patients had mutations in other genes including BRAF, DNMT3A, FLT3, IDH1, IDH2, JAK2, KRAS, and NPM1. This mutation frequency was not significantly different from t-MN (10/62 vs 10/39, $p=0.3070)$.

\section{TP53 mutation correlated with clinical parameters and cytogenetics}

t-MDS/AML with TP53 mutation showed a significantly lower mean corpuscular volume (MCV) (median, 90 vs. 93, $p=0.0076$ ) and platelet counts (median, $32 \times 10^{9}$ vs. $\left.42 \times 10^{9} / \mathrm{L}, \quad p=0.0393\right)$ compared with $\mathrm{t}-\mathrm{MN}$ without TP53 mutation. There were no significant differences in demographics, hemoglobin level, leukocytes, and absolute neutrophil counts, and prior cytotoxic therapy between patients with mutated or wild-type TP53.

The distribution of cytogenetic data was significantly different between $\mathrm{t}-\mathrm{MN}$ with and without TP53 mutation. 
Overall, a higher cytogenetic risk was more common in tMN with TP53 mutation $(p<0.0001)$, particularly, a complex karyotype $(p<0.0001)$ (Table 2$)$. In t-MDS/AML with a diploid or a non-complex aberrant karyotype $(n=49)$, TP53 mutation was detected in only three (6.1\%) patients. TP53 mutations were associated with frequent $-5 /-5 q$ abnormalities $(71.8 \%$ vs. $26.9 \%, p<0.0001)$ but not $-7 /-7 q$ abnormalities $(p=0.2067)$. When all numerical and structural chromosomal abnormalities were counted, TP53 mutation was shown not only to correlate with a complex karyotype but also the total number of karyotypic abnormalities $(P<0.0001)$ (Figure $2 \mathrm{~A}, \mathrm{~B})$, in both $\mathrm{t}-\mathrm{MN}$ and $d e$ novo MDS/AML.

\section{Prognostic significance of TP53 mutations in t-MN}

We collected survival data with a median follow-up of 12.9 months including alive and dead patients (range, 0.1-198.3). After censoring patients who received stem cell transplant (SCT) at the time of the procedure, the median overall survival (OS) of all t-MDS/AML patients was 8.0 months (range, $0.1-32.7$ ). t-MDS/AML patients with TP53 mutation showed a significantly shorter median OS (6.1 months) compared with patients with wildtype TP53 (14.1 months) $(p<0.0001)$ (Figure 3A). Of patients with TP53 mutations, the OS was very similar between patients with $\mathrm{t}-\mathrm{MN}$ or de novo MDS/AML (6.2 months, range 0.2-79.7) ( $p=0.8197)$ (Figure 3B). The location (exons) or type (transition/transversion) of
TP53 mutations did not correlate with OS $(p=0.2922$ and $p=0.9209$, respectively). In univariate analysis, hemoglobin level $(<10 \mathrm{~g} / \mathrm{dL})$, and platelet count $(<50 \times$ $10^{9} / \mathrm{L}$ ), a complex karyotype and TP53 mutation were identified as significant hazards. In multivariate analysis, platelet count, male gender and TP53 mutation $(p=$ 0.002) remained to be independent hazards (Table 3).

\section{Discussion}

In this study, we show that $\mathrm{t}-\mathrm{MN}$ carry a high frequency of TP53 mutation than their de novo counterparts. However, the mutation allele frequency, nucleotide alterations, and mutation patterns in TP53 are not different between MDS/AML patients with or without a history of cytotoxic exposure. TP53 mutation in t-MN is an independent risk for an inferior outcome.

In this cohort of $108 \mathrm{t}-\mathrm{MN}$ patients, TP53 mutation was detected in $37 \%$ of patients. This frequency of TP53 mutations was comparable to a frequency of $20 \% \sim 40 \%$ reported by others $[15,17,19]$. Most mutations were missense mutations and clustered in the DNA-binding domain (exons 5-8). The most common mutation type was C:G to T:A substitution (43.9\%), and this frequency was similar to the data reported by Wong and colleagues of approximately 50\% [18]. The most frequent mutated locus in t-MN was codon 248, which is one of the most frequently mutated codons (reported in COSMIC (http://cancer.sanger.ac.uk)) as well as in IARC

Table 2 Comparison between therapy-related myeloid neoplasm with and without TP53 mutation

\begin{tabular}{|c|c|c|c|}
\hline & Mutated TP53 $(n=40)$ & Wild-type TP53 $(n=68)$ & $p$ value \\
\hline Age, years, median (range) & $66(23-87)$ & $68(18-82)$ & $0.6491^{*}$ \\
\hline Male: female & $22: 18$ & $40: 28$ & 0.8405 \\
\hline \multicolumn{4}{|l|}{ Prior therapy } \\
\hline Chemotherapy only & 21 & 40 & 0.5518 \\
\hline Radiation only & 2 & 6 & 0.7082 \\
\hline Combined chemoradiation & 17 & 22 & 0.2972 \\
\hline Hemoglobin, g/L, median (range) & $91(66-1128)$ & $98(60-139)$ & $0.1775^{*}$ \\
\hline Mean corpuscular volume, median (range) & $90(70-107)$ & $93(79-116)$ & $0.0076^{*}$ \\
\hline White blood cell, $\times 10^{9} / \mathrm{L}$, median (range) & $3.0(0.3-41)$ & $3.0(0.6-93.9)$ & $0.5314^{*}$ \\
\hline Absolute neutrophil count, $\times 10^{9} / \mathrm{L}$, median (range) & $0.8(0-9.2)$ & $1.1(0-30.62)$ & $0.2223^{*}$ \\
\hline Platelet, $\times 10^{9} / \mathrm{L}$, median (range) & $32(5-394)$ & $42(7-364)$ & $0.0393^{*}$ \\
\hline Cytogenetic data & & & $<0.0001$ \\
\hline Diploid & 0 & 16 & \\
\hline Non-complex $(<3)$ & 3 & 30 & \\
\hline Complex ( $\geq 3)$ & 36 & 20 & \\
\hline Not available & 1 & 2 & \\
\hline Chromosome 5 aberrations & 28 & 14 & $<0.0001$ \\
\hline Chromosome 7 aberrations & 18 & 23 & 0.2067 \\
\hline Number of abnormalities, median (range) & $7(1-22)$ & $1(0-22)$ & $<0.0001^{*}$ \\
\hline
\end{tabular}

Asterisk (*) denotes $p$ value was calculated by Mann-Whitney $U$ test. All the others were calculated by Fisher's exact test. 

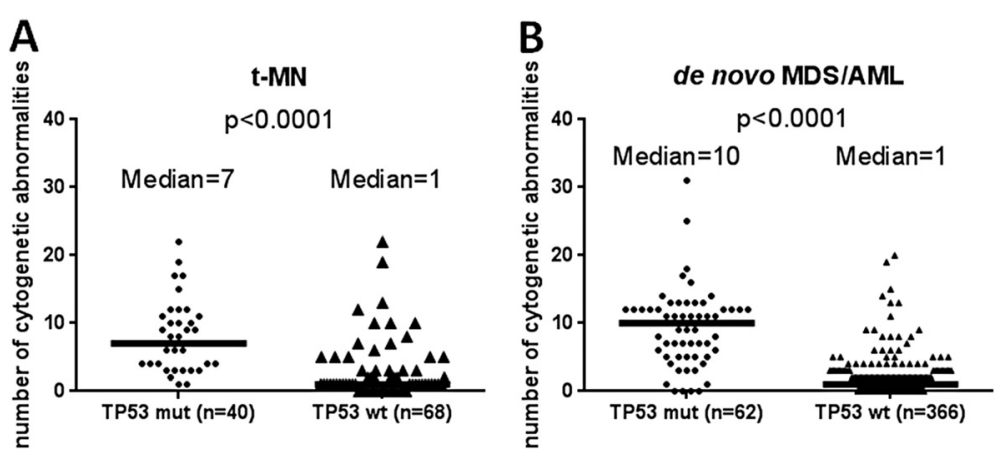

Figure 2 Number of cytogenetic abnormalities with respect to TP53 mutation in therapy-related myeloid neoplasm (A) and in de novo myelodysplastic syndromes/acute myeloid leukemia (MDS/AML) (B). TP53 mut, cases with TP53 mutation; TP53 wt, cases with wild-type TP53.

TP53 database (R17, November 2013). Mutations in this codon of TP53 have been reported in colorectal cancer, laryngeal squamous cell carcinoma, prostate cancer, breast cancer, and ovarian cancer [20].

We show that the pattern of TP53 mutation and mutational allelic frequency in $\mathrm{t}-\mathrm{MN}$ are similar to that in de novo MDS/AML. The lack of unique features of mutation in TP53 gene between t-MN and de novo diseases indicates that cytotoxic therapy does not cause genomewide damage in TP53; rather the genetic insult to TP53 gene is similar in MDS/AML either secondary to cytotoxic therapy or occurring de novo. The former concept was supported by the study of Wong and colleagues [18] who showed that TP53 mutations detected at the diagnosis of t-AML were also found in mobilized blood leukocytes or bone marrow 3-6 years prior to the diagnosis of $\mathrm{t}-\mathrm{MN}$ in the same patients. They proposed a model of t-AML harboring clonal TP53 mutation that somatic TP53 mutations are present in the hematopoietic stem cells (HPSC) of some healthy individuals; and these HPSC may expand under the selective pressure of chemotherapy. The findings derived from this large patient cohort in this study endorse this disease model.
Our group has shown that using NGS methods, the mutational profile t-MDS and t-AML, are different [9]. The overall mutation frequency and the number of involved genes were significantly higher in $\mathrm{t}-\mathrm{AML}$ than $\mathrm{t}$ MDS. However, with respect to TP53, mutations were equally frequent in t-AML and t-MDS. In this study, with this expanded patient cohort, not only did we further confirm our previous observation but also showed that the allele frequency and mutation pattern of TP53 were nearly identical in t-MDS and t-AML. Interestingly, 5 of $11(45.5 \%)$ transversions detected in t-MDS were C:G > A:T substitution. This specific substitution was detected in 1 of 7 (14.3\%) transversions in de novo MDS and was not detected in t-AML or de novo AML. The prevalence of such specific mutations was reported to be significantly higher in smoking-associated lung cancers compared to lung cancers of non-smokers [21]. However, it was also reported that the frequencies of transitions versus transversions as well as the specific type of transitions/transversion of TP53 mutations in breast cancer patients differed by ethnic background and had no clinical significance [22]. It is uncertain at this point if this specific transversion was more prevalent in MDS
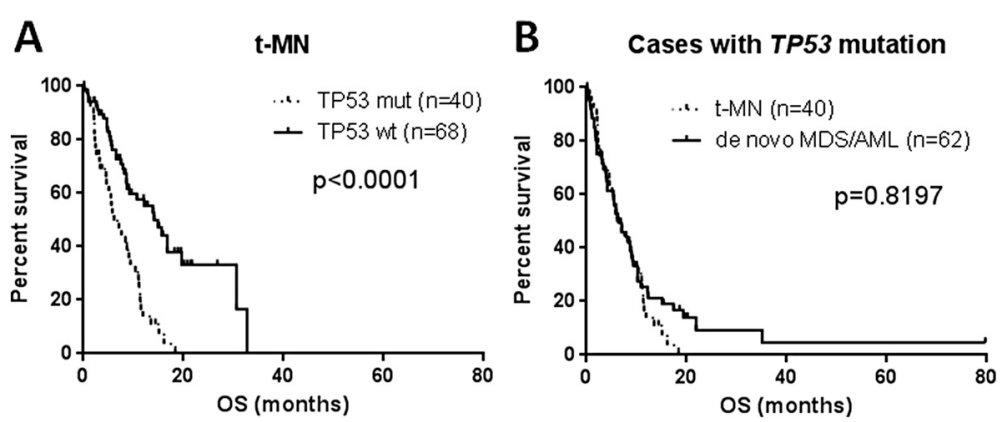

Figure 3 OS comparison. Overall survival (OS) comparison with respect to TP53 mutation in patients with therapy-related myeloid neoplasm (t-MN) (A). OS comparison between t-MN with TP53 mutation and de novo MDS/AML with TP53 mutation (B). TP53 mut, patients with TP53 mutation; TP53 wt, patients with wild-type TP53. 
Table 3 Overall survival of patients with therapy-related myeloid neoplasm by univariate and multivariate analysis

\begin{tabular}{|c|c|c|c|c|c|c|}
\hline \multirow[t]{2}{*}{ Variables } & \multicolumn{3}{|c|}{ Univariate analysis } & \multicolumn{3}{|c|}{ Multivariate analysis } \\
\hline & $\mathrm{HR}$ & $95 \% \mathrm{Cl}$ & $p$ value & $\mathrm{HR}$ & $95 \% \mathrm{Cl}$ & $p$ value \\
\hline Age (>60 years) & 1.001 & $0.572-1.753$ & 0.997 & 0.995 & $0.553-1.792$ & 0.988 \\
\hline Gender (man) & 0.708 & $0.438-1.143$ & 0.157 & 0.541 & $0.315-0.927$ & 0.025 \\
\hline $\operatorname{ANC}\left(<0.8 \times 10^{9} / \mathrm{L}\right)$ & 1.313 & $0.821-2.099$ & 0.256 & 0.820 & $0.482-1.392$ & 0.462 \\
\hline BM blast $(\geq 5 \%)$ & 1.633 & $0.941-2.836$ & 0.081 & 1.580 & $0.853-2.924$ & 0.145 \\
\hline Hemoglobin $(<100 \mathrm{~g} / \mathrm{L})$ & 1.946 & $1.153-3.286$ & 0.013 & 1.711 & $0.950-3.081$ & 0.073 \\
\hline Platelet $\left(<50 \times 10^{9} / \mathrm{L}\right)$ & 2.181 & $1.291-3.683$ & 0.004 & 2.023 & $1.153-3.549$ & 0.014 \\
\hline Complex karyotype & 2.878 & $1.694-4.888$ & $<0.001$ & 1.150 & $0.546-2.425$ & 0.713 \\
\hline TP53 mutation & 2.971 & $1.823-4.841$ & $<0.001$ & 2.958 & $1.476-5.929$ & 0.002 \\
\hline
\end{tabular}

Abbreviations: $\mathrm{HR}$, hazard ratio; $\mathrm{Cl}$, confidence interval; $\mathrm{ANC}$, absolute neutrophil count; $\mathrm{BM}$, bone marrow. $p$ value $<0.05$ is demonstrated in italics.

over AML. Nevertheless, our findings in t-MDS vs tAML provide further support to the model that TP53 mutation is an early event in pathogenesis of $\mathrm{t}-\mathrm{MN}$, and additional molecular genetic events, particularly mutations in class I genes, likely provide proliferative advantage in cases of t-AML.

Similar to what has been reported previously $[17,19,23]$, TP53 mutations were highly associated with a complex karyotype and frequent deletions involving chromosome 5 in t-MN. In this study, we further showed that TP53 mutation correlated with a higher number of structural and numerical chromosomal abnormalities in $\mathrm{t}-\mathrm{MN}$. On the other hand, TP53 mutations were identified in approximately $5 \%$ of cases with a normal or a non-complex karyotype in $\mathrm{t}-\mathrm{MN}$. It is likely that p53 dysfunction leads to genome instability and facilitates cytogenetic complexity. It is noteworthy that approximately $40 \%$ of $\mathrm{t}-\mathrm{MN}$ cases with a complex karyotype had no TP53 mutations, suggesting that other factors, probably multiple cytotoxic insults, may contribute to karyotypic complexity. We also showed that TP53 mutation in t-MN predicts a shorter overall survival in t-MN, and the risk of TP53 mutation is an independent adverse risk factor. In contrast, a complex karyotype failed to show its independent prognostic value when it was co-analyzed with other confounding factors including TP53 mutation status.

\section{Conclusions}

In summary, $\mathrm{t}$-MDS and $\mathrm{t}$-AML both harbor a high frequency of TP53 mutations, significantly higher than their de novo counterpart. However, the mutation type, pattern, distribution of mutated loci, and mutational allelic frequency in TP53 are neither different between therapyrelated and de novo MDS/AML nor between t-MDS and t-AML. These findings support the recent model proposed by Wong and colleagues that TP53 mutation occurs at a very early stage of leukemogenesis of $\mathrm{t}-\mathrm{MN}$, but other factors likely contribute to further development of clinically and histopathologically evident t-MN. Overall, TP53 mutation in t-MN is strongly associated with a complex karyotype as well as the number of karyotypic abnormalities. TP53 mutation predicts a poorer survival and is an independent adverse risk factor in patients with $\mathrm{t}-\mathrm{MN}$.

\section{Methods \\ Patients}

We collected 108 consecutive patients with therapyrelated MDS/AML from October 2012 through January 2014 at the University of Texas MD Anderson Cancer Center. Clinical, hematological, and cytogenetic data were collected by searching the electronic medical record. The types of primary malignant or non-malignant diseases for which cytotoxic therapy was administered were also collected. Brachytherapy, radioisotopes, and radiation therapy in patients in whom the field did not include active hematopoietic bone marrow were not considered as radiation therapy. All cases were collected consecutively and classified according to the World Health Organization (WHO) classification system. A previously published patient cohort consisting of 428 patients with de novo MDS/ AML was included for comparison [9]. This study was conducted in accord with the Declaration of Helsinki and was approved by the IRB at the University of Texas MD Anderson Cancer Center in Houston, TX, USA.

\section{TP53 sequencing}

TP53 sequencing was performed in all patients using a clinically validated 53 -gene panel or a 28 -gene panel assay. The 53-gene panel covers exons (codons) 2, 4-8, and $10(1-12,69-112,126-253,267-206$, and 332-342) and the 28-gene panel covers exons (codons) 4-10 (41224 and 234-367). Briefly, genomic DNA (gDNA) was extracted from bone marrow aspirate or peripheral blood of each case using an Autopure extractor (Qiagen, Valencia, CA, USA). A sequencing library was prepared using $250 \mathrm{ng}$ of DNA template and either 53- or 28-gene panel. The sequencing library was purified using AMPure magnetic beads (Agencourt, Brea, CA, USA) and then 
subjected to MiSeq sequencer (Illumina Inc., San Diego, CA, USA) [24]. A minimum quality score of AQ30 is required for a minimum of $75 \%$ of bases sequenced ensuring high-quality sequencing results. Variant calling was performed with Illumina MiSeq Reporter Software 1.3.17 using human genome build 19 (hg 19) as a reference and sequencing reads were aligned using the Integrative Genomics Viewer (IGV, Broad Institute, MA, USA) [25]. For clinical reporting, a sequencing coverage of $250 \times$ (bidirectional true paired-end sequencing) and a variant frequency of $5 \%$ in a background of wild-type TP53 were used as cutoffs.

\section{Cytogenetic analysis}

Conventional cytogenetic analysis was performed using standard methods as described previously [26]. Twenty metaphases were analyzed, and the results were reported using the current International System for Human Cytogenetic Nomenclature [27]. Only karyotypes with adequate metaphases for analysis were included, except in some cases where lesser numbers of metaphases were available, fluorescence in situ hybridization (FISH) was performed to confirm clonal cytogenetic abnormalities. For MDS patients, the cytogenetic risk was stratified according to the IPSS [28]; and for AML patients, the risk was categorized by the revised cytogenetic classification proposed by the UKMRC [29].

\section{Statistical analysis}

For continuous variables, data were reported as a median and range. For nominal variables, data were reported as the number of patients if not otherwise specified. Fisher's exact test and the Mann-Whitney $U$ test were used for categorical variables and for continuous variables, respectively. OS was calculated from the day of diagnosis to the last follow-up. For patients who received hematopoietic stem cell transplant (HSCT), survival was censored at the day of the procedure. Distributions of OS were estimated by Kaplan and Meier curves and survival differences were evaluated using the log-rank test. All differences with $p<$ 0.05 were considered to be statistically significant (twotailed). GraphPad Prism 6.0 (La Jolla, CA, USA) and SPSS V22 (Armonk, NY, USA) were used for statistical analyses.

\section{Competing interests}

The authors declare that they have no competing interests.

\section{Authors' contributions}

CYO and SAW conceived the idea. CYO, JP, and MG carried out data collection. CYO, KPP, MJR, JP, GT, MG, RS, and RL performed data analysis. GGM and HMK provided patient material. CYO, KHY, LMM and SAW wrote the manuscript. SAW supervised the study. All authors read and approved the final manuscript.

\section{Author details}

'Department of Hematopathology, The University of Texas MD Anderson Cancer Center, 1515 Holcombe Boulevard, Houston, TX, USA. ${ }^{2}$ Department of
Leukemia, The University of Texas MD Anderson Cancer Center, 1515 Holcombe Boulevard, Houston, TX, USA. ${ }^{3}$ Department of Hematology, Central South University Xiangya Hospital, Changsha, China.

Received: 18 February 2015 Accepted: 20 April 2015

Published online: 08 May 2015

\section{References}

1. Vardiman JW, Arber DA, Brunning RD, Larson RA, Matutes E, Baumann I, et al. Therapy-related myeloid neoplasms. In: Swerdlow SH et al., editors. WHO classification of tumours of haematopoietic and lymphoid tissues. 4th ed. Lyon: International Agency for Research on Cancer (IARC); 2008. p. 127-9.

2. Singh ZN, Huo D, Anastasi J, Smith SM, Karrison T, Le Beau MM, et al. Therapy-related myelodysplastic syndrome: morphologic subclassification may not be clinically relevant. Am J Clin Pathol. 2007;127:197-205.

3. Ok CY, Hasserjian RP, Fox PS, Stingo F, Zuo Z, Young KH, et al. Application of the international prognostic scoring system-revised in therapy-related myelodysplastic syndromes and oligoblastic acute myeloid leukemia. Leukemia. 2014;28:185-9.

4. Bhatia S. Therapy-related myelodysplasia and acute myeloid leukemia. Semin Oncol. 2013;40:666-75.

5. Greco M, D'Alò F, Scardocci A, Criscuolo M, Fabiani E, Guidi F, et al. Promoter methylation of DAPK1, E-cadherin and thrombospondin-1 in de novo and therapy-related myeloid neoplasms. Blood Cells Mol Dis. 2010;45:181-5

6. Voso MT, D'Alò F, Greco M, Fabiani E, Criscuolo M, Migliara G, et al. Epigenetic changes in therapy-related MDS/AML. Chem Biol Interact. 2010;184:46-9.

7. Smith SM, Le Beau MM, Huo D, Karrison T, Sobecks RM, Anastasi J, et al. Clinical-cytogenetic associations in 306 patients with therapy-related myelodysplasia and myeloid leukemia: the University of Chicago series. Blood. 2003;102:43-52.

8. Zhou Y, Tang G, Medeiros LJ, McDonnell TJ, Keating MJ, Wierda WG, et al. Therapy-related myeloid neoplasms following fludarabine, cyclophosphamide, and rituximab (FCR) treatment in patients with chronic lymphocytic leukemia/ small lymphocytic lymphoma. Mod Pathol. 2012;25:237-45.

9. Ok CY, Patel KP, Garcia-Manero G, Routbort MJ, Fu B, Tang G, et al. Mutational profiling of therapy-related myelodysplastic syndromes and acute myeloid leukemia by next generation sequencing, a comparison with de novo diseases. Leuk Res. 2014;39:348-54.

10. Xu-Monette $Z Y$, Medeiros $L$, Li Y , Orlowski RZ, Andreeff M, Bueso-Ramos $C E$, et al. Dysfunction of the TP53 tumor suppressor gene in lymphoid malignancies. Blood. 2012;119:3668-83.

11. Olivier $M$, Hollstein $M$, Hainaut P. TP53 mutations in human cancers: origins, consequences, and clinical use. Cold Spring Harb Perspect Biol. 2010;2:a001008.

12. Kulasekararaj AG, Smith AE, Mian SA, Mohamedali AM, Krishnamurthy P, Lea NC, et al. TP53 mutations in myelodysplastic syndrome are strongly correlated with aberrations of chromosome 5, and correlate with adverse prognosis. Br J Haematol. 2013;160:660-72.

13. Bejar R, Stevenson K, Abdel-Wahab O, Gailii N, Nilsson B, Garcia-Manero G, et al. Clinical effect of point mutations in myelodysplastic syndromes. N Engl J Med. 2011;364:2496-506.

14. Bejar R, Levine R, Ebert BL. Unraveling the molecular pathophysiology of myelodysplastic syndromes. J Clin Oncol. 2011;29:504-15.

15. Ben-Yehuda D, Krichevsky S, Caspi O, Rund D, Polliack A, Abeliovich D, et al. Microsatellite instability and p53 mutations in therapy-related leukemia suggest mutator phenotype. Blood. 1996;88:4296-303.

16. Christiansen DH, Andersen MK, Pedersen-Bjergaard J. Mutations with loss of heterozygosity of p53 are common in therapy-related myelodysplasia and acute myeloid leukemia after exposure to alkylating agents and significantly associated with deletion or loss of 5q, a complex karyotype, and a poor prognosis. J Clin Oncol. 2001;19:1405-13.

17. Shih AH, Chung SS, Dolezal EK, Zhang SJ, Abdel-Wahab OI, Park CY, et al. Mutational analysis of therapy-related myelodysplastic syndromes and acute myelogenous leukemia. Haematologica. 2013;98:908-12.

18. Wong TN, Ramsingh G, Young AL, Miller CA, Touma W, Welch JS, et al. Role of TP53 mutations in the origin and evolution of therapy-related acute myeloid leukaemia. Nature. 2014;518:552-5.

19. Pedersen-Bjergaard J, Andersen MK, Andersen MT, Christiansen DH. Genetics of therapy-related myelodysplasia and acute myeloid leukemia. Leukemia. 2008;22:240-8. 
20. Petitjean A, Mathe E, Kato S, Ishioka C, Tavtigian SV, Hainaut P, et al. Impact of mutant p53 functional properties on TP53 mutation patterns and tumor phenotype: lessons from recent developments in the IARC TP53 database. Hum Mutat. 2007;28:622-9.

21. Pfeifer GP, Denissenko MF, Olivier M, Tretyakova N, Hecht SS, Hainaut P. Tobacco smoke carcinogens, DNA damage and p53 mutations in smoking-associated cancers. Oncogene. 2002;21:7435-51.

22. Nagai MA, Schaer Barbosa H, Zago MA, Araújo Silva Jr W, Nishimoto IN Salaorni S, et al. TP53 mutations in primary breast carcinomas from white and African-Brazilian patients. Int J Oncol. 2003;23:189-96.

23. Bowen D, Groves MJ, Burnett AK, Patel Y, Allen C, Green C. TP53 gene mutation is frequent in patients with acute myeloid leukemia and complex karyotype, and is associated with very poor prognosis. Leukemia. 2009;23:203-6.

24. Junemann S, Sedlazeck FJ, Prior K, Albersmeier A, John U, Kalinowski J, et al. Updating benchtop sequencing performance comparison. Nat Biotechnol. 2013;31:294-6.

25. Robinson JT, Thorvaldsdóttir H, Winckler W, Guttman M, Lander ES, Getz G, et al. Integrative genomics viewer. Nat Biotechnol. 2011;29:24-6.

26. Khoury JD, Sen F, Abruzzo LV, Hayes K, Glassman A, Medeiros $L$. Cytogenetic findings in blastoid mantle cell lymphoma. Hum Pathol. 2003;34:1022-9.

27. Shaffer LG, McGowan-Jordan J, Schmid M. An international system for human cytogenetic nomenclature. In: Shaffer LG, McGowan-Jordan J, Schmid M, editors. Basel: Karger; 2013.

28. Greenberg P, Cox C, LeBeau MM, Fenaux P, Morel P, Sanz G, et al. International scoring system for evaluating prognosis in myelodysplastic syndromes. Blood. 1997:89:2079-88.

29. Grimwade D, Hills RK, Moorman AV, Walker H, Chatters S, Goldstone AH, et al. Refinement of cytogenetic classification in acute myeloid leukemia: determination of prognostic significance of rare recurring chromosomal abnormalities among 5876 younger adult patients treated in the united kingdom medical research council trials. Blood. 2010;116:354-65.

\section{Submit your next manuscript to BioMed Central and take full advantage of:}

- Convenient online submission

- Thorough peer review

- No space constraints or color figure charges

- Immediate publication on acceptance

- Inclusion in PubMed, CAS, Scopus and Google Scholar

- Research which is freely available for redistribution 